\title{
A multiple species approach to sequential learning: Are you a man or a mouse?
}

\author{
JAMES D. ROWAN \\ Wesleyan College, Macon, Georgia \\ STEPHEN B. FOUNTAIN \\ Kent State University, Kent, Ohio \\ and \\ SHANNON M. A. KUNDEY and CHRISSY L. MINER \\ Wesleyan College, Macon, Georgia
}

\begin{abstract}
We have developed a method for studying list learning in animals and humans, and we use variants of the task to examine list learning in rats, mice, and humans. This method holds several advantages over other methods. It has been found to be easily learned without lengthy pretraining. The data gathered with this procedure provide a measure of correct response rates, of incorrect responses and the locations of these responses, and of response latency on a trial-by-trialbasis. We have examined mouse, rat, and human list acquisition of patterns ranging from 12 to 48 items in length. This procedure has also been used to examine many aspects of list learning, such as the effects of the placement of phrasing cues that are either consistent or inconsistent with the structure of the list in rats and mice, the effects of phrasing cues of differing modalities in mice, the sensitivity of subjects to violations of list structure in rats, subjects' abilities to "chunk" from nonadjacent serial positions in structured lists in rats, and subjects' sensitivity to serial patterns with multiple levels of hierarchical organization. The procedure has also been used to examine the effects of drugs on sequential learning.
\end{abstract}

All living organisms have to deal with a continuous stream of stimuli. Thus, most behavior is, in essence, a list learning/memory task. For a behavior to be successful in most situations, the animal must not only learn a group of responses, but must also learn to produce these responses in a specific temporal order. A rat's learning that two turns in one direction and one turn in another in a laboratory maze must be made in order to obtain food benefits only if the turns are made in the correct order. Similarly, remembering the seven digits of someone's phone number is beneficial only when they are dialed in the correct order. There are many instances such as these in which the correct ordering of a series of responses will determine the success of the behavioral sequence. This fact was pointed out by Lashley (1951), who was one of the first modern psychologists to suggest that learning complex lists of responses cannot be adequately explained by stimulus-response chaining theories. Instead, he proposed that organisms actively employ central or-

This work was supported by National Institute of Mental Health Grant MH48402 and in part by National Institutes of Health Extramural Associates Program Grant HD32814. We thank Barbara Rowan for her aid in editing this paper. The experiments reported in this paper were conducted in compliance with the current laws of the United States. Correspondence should be addressed to J. D. Rowan, Department of Psychology, Wesleyan College, 4760 Forsyth Rd., Macon, GA 31210 (e-mail: jrowan@wesleyancollege.edu). ganization to generate a series of responses to more efficiently learn sequences in serial learning.

Since Lashley's proposal in 1951, the topic of serial order in learning has received much attention. Many studies have been conducted to examine serial learning processes in humans and have often employed tasks that required human subjects to learn sequences of letters or digits. A variety of evidence has indicated that, when human subjects learn organized sequences of letters or digits (i.e., serial patterns), they are sensitive to pattern order. The processes involved in serial pattern learning have also been examined in other species, including rats, pigeons, nonhuman primates, and songbirds. In general, the evidence indicates that animals, like humans, are sensitive to pattern order.

The idea that animals are sensitive to the structure of patterns is well supported. For instance, rats have been trained to anticipate sequences of varying food quantities in runway maze and operant tasks and sequences of quantities of brain-stimulation reward (BSR; Fountain \& Annau, 1984). Rats have also been trained to anticipate the sequential positions of flashing lights in an array (Fountain, 1990).

\section{Evolution of a Procedure}

In earlier studies examining animals' abilities to learn structured patterns, or serial patterns, Hulse and Dorsky (1977) demonstrated that rats trained on a runway task to 
run for various quantities of food pellets could use the structure of the pattern to learn the sequence faster if its elements were organized by some simple rule. Rats were tested in a runway and received different quantities of food pellets in the goal box at the end of each run. In one experiment, the goal box contained $14,7,3,1$, or 0 food pellets. Subjects were given sets of five runs in the runway. In each series of five runs, one group of rats received 14 pellets on the first run, 7 on the second, 3 on the third, 1 on the fourth, and 0 on the last run (the sequence was 14-7-3-1-0, a monotonically decreasing pattern). Response latency was used to measure response to the various food quantities. As the rats learned the sequence, they had to anticipate the amount of food at the end of the runway as indicated by their running times. The interesting result was that rats came to run much more slowly on the last run of each series (i.e., for the 0-pellet quantity). Hulse and Dorsky attributed the difference in running speed on the last run to learning. A control group (nonmonotonic pattern) received the same food quantities as the experimental group but in a different sequential order, which could not be easily expressed by a rule (14-1-37-0 pellets). When the running times of the rats in the monotonic group were compared with those of the nonmonotonic group, the rats in the latter group learned the last element more slowly than did the rats in the monotonic group.

In early studies that examined human serial-pattern learning, subjects had to learn longer patterns with more complex structure. For example, Restle and Burnside (1972) had human subjects track an array of six flashing indicator lights by pressing buttons associated with the lights. They found that the subjects' pattern of errors indicated that they were sensitive to the formal structure of the rule-based pattern of the flashing indicator lights. In the first experiment, their subjects were required to track the indicator lights (numbered 1 to 6 from left to right) as they flashed in the sequence 1-2-3-4-6-6-6-6-6-2-32-3-5-4-3. Examination of the structure of the pattern shows that the pattern can be broken into four chunks. The first chunk, 1-2-3-4, can be described by a rule that defines the next element by a move one to the right, or the " +1 " rule. The second chunk, 6-6-6-6-6, is described by a repeat rule on the same key. The third chunk, 2-3$2-3$, can be described by a trill or alternate rule. The last chunk, 5-4-3, can be described by a move one to the left, or the " -1 " rule. Subjects produced the most errors at the beginning of the chunks and relatively few errors within chunks, as the rule-learning theory predicts. Restle and Burnside reported that two specific errors were most common at the boundaries of chunks. The first was an error of anticipation, where the subject applied the rule for the next chunk too early. For example, in the second chunk, 6-6-6-6-6, the subjects would, on the fifth element of this chunk, respond " 2 ," anticipating the first element of the third chunk. The other error was one of extrapolation, where the subjects overextended a rule used in the chunk, producing predictable errors on the first element of the next chunk.
It was obvious that the methods used to study human serial pattern learning were more versatile with respect to the length and complexity of the patterns that could be presented to the subject to learn. This fact challenged researchers to develop new tasks for animals that corresponded better to the ones used with humans. In an operant procedure analogous to the one used with humans by Restle and Brown (1970), Fountain (1990) trained rats to track the locations of flashing indicator lights in a horizontal six-light array by pressing levers in a corresponding array below the lights (Figure 1). In one experiment, rats were presented the sequence 1-2-3-4-6-6-6-6-6-4-34-3-5-4-3 (levers were numbered 1 to 6 from left to right). The numbers indicate reinforced leverpresses for each trial. The pattern tracking in this procedure was very rapid because lights in the horizontal array flashed on and off every second with a 1 -sec intertrial interval (ITI) and because responses were reinforced with brief pulses of BSR.

Like Restle and Brown (1970), by using the structurally equivalent pattern with human subjects, Fountain (1990) found that rats' errors and omitted responses were more likely and correct responses were less likely at the beginning of structural chunks. In fact, the distribution of errors produced by rats paralleled the results obtained by Restle and Burnside (1972) with human subjects.

Further refinements in the procedure were made to correct some of the shortcomings of the Restle and Brown (1970) human task and the Fountain (1990) rat task. One of the major drawbacks of these procedures was that a linear array of stimulus choices was used. Once a subject had reached the end of the array, there was no possibility of the sequence's continuing beyond that point. If a chunk of information ended with the first or last manipulum in the array, there was no choice for the subject to overextend the chunk and produce errors. When two extra levers were added to the chamber to allow for overextension errors at the beginning and ends of the array, the rats still did not produce the expected errors. For the rats, the end walls made very salient cues for the positions of the chunk boundaries and the beginning and end of the linear array of levers. Also, because the manipuli were in a fixed position in the chamber, or always present to respond to, physical momentum of the subject could contribute to the production of the response as much as the subject's choice, especially in the case of the Fountain task. Animals responding in one direction may have simply activated the next lever in the array because they were not able to change direction. However, the last criticism cannot account for the fact that the rats showed shorter latencies in responding and increased accuracy (Fountain, 1990). If physical momentum was entirely responsible for the Fountain results, it would have been expected that the "overruns" would have become more prevalent as the speed of responding increased, and not less prevalent.

\section{Development of the Eight-Choice Circular Array Procedure}

Because of the limitations of a linear array approach, a new procedure was developed for examining serial pat- 


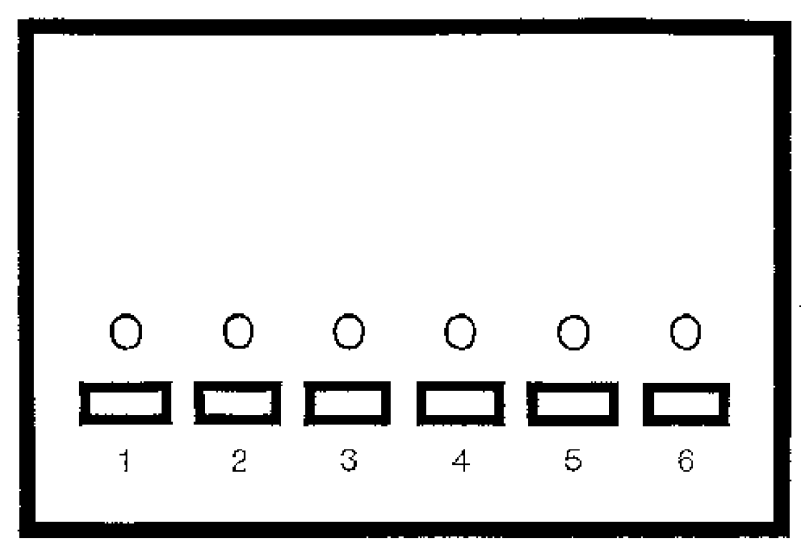

Figure 1. Schematic representation of the six-lever linear array used in Fountain (1990). Digits represent the positions of the levers used in the formation of the response patterns.

tern learning in rats. This procedure was eventually adapted for humans and mice. Though the apparatus and procedures in the numerous variants of this procedure differ, there are some essential commonalities. The critical feature of this procedure is that elements of a pattern are drawn from a circular array of stimuli. In the case of rats and mice, the subjects can respond to manipuli that are individually located on each wall of an operant chamber. For humans, the response set consists of a circular arrangement of circles on a computer screen. The patterns are composed of the positions of the different response items. This means that no end walls of an operant chamber or end keys on a human task can be used to discriminate one pattern element from another. The patterns generated can be continuous-that is, they can be composed of the endless combination of the manipuli to form patterns of any length and complexity. This is a choice procedure; thus, the data obtained should be less affected by physical momentum or other contaminants related to the response speed of the subject. The data gathered with this procedure provide (1) a measure of correct response rates, (2) a measure of incorrect responses and the locations of these responses, and (3) a measure of response latency on a trial-by-trial basis. Subjects can receive numerous patterns each day without interruption between patterns. The limitation of pattern length and pattern number used in a testing session is set only by the maximum amount of reinforcement for which a subject will work before becoming satiated. Although the procedure has only been used to examine serial pattern learning in humans, mice, and rats, it could be easily adapted for use with any species that generates the required number of responses needed for each testing session.

\section{Variations of the Eight-Choice Task}

The earliest research using the circular array examined rats' ability to learn structurally simple patterns of positions within the chamber. The test chamber used was octagonal in shape with walls that were $15 \mathrm{~cm}$ wide and $30 \mathrm{~cm}$ tall (see Figure 2). The chamber measured approximately $40 \mathrm{~cm}$ between parallel walls and was composed of clear Plexiglas walls and a floor of hardware cloth. A retractable response lever was centered on each wall $5.0 \mathrm{~cm}$ above the floor. The rats in the experiment were connected to a stimulator by way of a flexible cord and a commutating device centered in the ceiling of the chamber. Experiments were controlled from an adjoining room with the use of a microcomputer and interface (interface and Med-State Software, Med Associates, Inc., Fairfield, VT).

A discrete trial, two-choice procedure with correction was used. At the beginning of each trial, two levers were inserted into the chamber. The two levers, except for those in the first trial of the testing session, were located on either side of the last correct lever. When the rat produced a response on the correct lever, both levers were retracted from the chamber, and a pulse of BSR was administered. When the rat produced an incorrect response, the incorrect lever was retracted, and no reward was administered until the rat pressed the correct lever. This correction procedure ensured that the rats received feedback regarding the correct lever on each trial. An ITI of $1 \mathrm{sec}$ was imposed except where phrasing conditions called for longer intervals. The rats typically required shaping in this procedure for 1 day or, at most, 2 days. Those that failed to acquire the task within 2 days were excluded from the experiment. In one experiment (Fountain \& Rowan, 1995b), rats received either patterns with a perfect, simply expressed structure or nearly identical patterns in which the last element violated the pattern

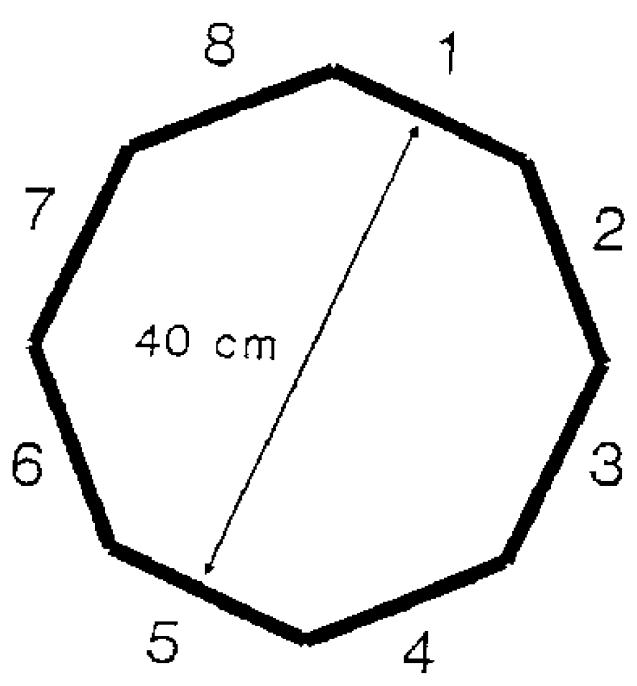

Figure 2. Schematic representation of the octagonal operant chamber (top view). Digits represent clockwise positions of the response manipuli (either retractable response levers or nosepoke infrared emitter/detector units). 
structure. All patterns were phrased by 3 -sec pauses between every third element. In one pattern, the cues grouped the pattern into runs,

\section{2 ,}

where the integers refer to the clockwise positions of the eight levers and spaces indicate 3 -sec pauses that separate the chunks. All other ITIs between elements were $1 \mathrm{sec}$. The violation pattern that corresponded to the perfect runs pattern was identical except that the last element was an 8 instead of a 2 :

$$
123234345456567678781818 .
$$

Another pattern was grouped by 3 -sec pauses into threeelement trills that could be described by an alternate (back and forth) rule (Fountain \& Rowan, 1995b):

$$
121232343454565676787818 .
$$

The violation group that corresponded to the trills pattern was identical, with the exception that the last element of the pattern was a 2 instead of an 8:

$$
121232343454565676787812 .
$$

(Note that Lever 1 was immediately to the right of Lever 8 , so that 812 and 818 were continuous runs and trills, respectively.)

High error rates were observed on the violation element for both violation runs and violation trills patterns. No comparable errors were observed for the perfect runs and trills patterns. For both violation conditions, however, the rats did eventually learn the position of the violation element and made the correct response. The results support the view that rats initially use a response strategy that allows for the production of the most correct responsesin this case, a rule-based strategy - and only later learn the elements that are exceptions to the rule. The results are best explained by the hypothesis that a rule was generated that best expressed the pattern's structure and then a rote strategy was used to learn the violation element.

Other experiments also examined mice's abilities to learn similar patterns (Fountain, Krauchunas, \& Rowan, 1999). The test chamber used was also octagonal in shape and was composed of clear Plexiglas walls with a floor of hardware cloth. This chamber was half the size of the rat test chamber, with 7.5-cm-wide and 15-cm-tall walls and approximately $20 \mathrm{~cm}$ between parallel walls. Centered on each wall, $2 \mathrm{~cm}$ off the floor, was a nosepoke receptacle with a light located on the back of it. This receptacle also contained a small line that delivered water reinforcement. Nosepokes into the receptacle activated an infrared emitter/detector that registered the response. As in Fountain and Rowan (1995b), the experiments were controlled from an adjoining room by using a microcomputer and interface (interface and Med-State Software, Med Associates, Inc., Fairfield, VT).

Each trial began with all lights in all eight receptacles turned on. The mouse selected one of the receptacles. A correct response resulted in water reinforcement. All lights were then turned off and then back on to signal the start of the next trial. If the mouse made an incorrect re- sponse, all the lights went off except for the correct one. A correct response from the mouse resulted in water reinforcement. Again, all lights went off and then came back on to signal the start of the next trial.

After pretraining, mice were assigned to four groups and received either a pattern with no structural flaws (perfect pattern) or the same pattern with a single violation element (violation pattern) as the final element of the sequence:

perfect pattern: 123234345456567678781812 , and

violation pattern: 123234345456567678781818 ,

in which the digits indicate the clockwise position of the correct response on successive trials. These patterns were presented with and without temporal phrasing cues. This resulted in four groups: perfect unphrased (PU), perfect phrased (PP), violation unphrased (VU), and violation phrased (VP). One should note that these patterns, except for the different phrasing conditions, are the same as the runs patterns used in the Fountain and Rowan (1995b) experiment, in which rats were subjects.

The general finding was that mice, like the rats in the Fountain and Rowan (1995b) experiment, found the chunk boundaries and violation elements more difficult to learn than the elements within the chunks. Their performance, however, seemed to be impaired by the temporal phrasing or visual and auditory phrasing, as was shown in Experiment 2. This differs from findings with rats when this procedure was used (Fountain et al., in press), in that rats' performances improved when temporal phrasing cues were consistent with the structure of the pattern.

Modifications of the procedure have also been employed to examine human serial pattern learning. Fountain and Rowan (1995a) compared rats' and humans' performance on similar variants of the original task. The human variation of this task requires students to respond on a computer. Subjects first read a simple set of instructions that was presented on the computer screen. They were informed that they would see a set of eight circles on the screen and that they were to use an arrow (cursor) key to move a smaller circular cursor to the circle of their choice. They were to then press the space bar to choose the circle. The instructions required the subjects to locate and use the right and left arrow keys and the space bar before the experiment began. Subjects were then informed that they would be given feedback as to the correctness of their choice. They were told not to be concerned if they made errors, to guess when necessary, and to follow the instructions in the box at the top of the screen if they forgot what to do. At the beginning of the testing session, eight circles (13 $\mathrm{mm}$ in diameter) appeared on the screen along with a message in the help box at the top of the screen instructing the subject to make a choice. The circles were equally spaced in a circular arrangement (opposite circles were $104 \mathrm{~mm}$ apart). Subjects moved the 
cursor to one of the circles and selected it by pressing the space bar. If the subject selected the correct circle, then "CORRECT" was displayed on the center of the computer screen during the ITI. If the subject selected the incorrect circle, the correct one remained displayed, and the other incorrect circles were removed. The subject was then given screen instructions for choosing the correct circle.

Likewise, the rat training procedure was identical to the Fountain and Rowan (1995b) task, except that all eight levers were inserted into the chamber for the rats to choose from, instead of only the two on either side of the last correct element. It was originally thought that it would be too difficult for the rats to learn the pattern if they had eight choices to choose from. This was found not to be the case. In fact, they learned the procedure at approximately the same rate as those in the earlier twochoice procedure.

Both groups of subjects in Fountain and Rowan (1995a) learned complex hierarchical patterns or linear patterns with elements containing the same pairwise associations as the hierarchical patterns but with the overall pattern structure disrupted. In three experiments, we compared rats' and humans' learning of patterns of increasing hierarchical complexity. Experiment 1 compared rats' and humans' performance in learning either a pattern that could be expressed with two rules and had perfect structure or the same pattern with the structure disrupted. The pairwise associations between the elements remained the same. In Experiment 2, we examined the effects of disrupting the structure in a pattern that had three rules to express its structure. In Experiment 3, we examined this phenomenon in a four-rule pattern. The error profiles generated by the subjects indicated that both humans and rats were using similar cognitive strategies in the learning of the patterns.

Other variations of the circular array procedure have also been developed and are currently in use. The octagonal maze procedure using the nosepoke as the operant response and water as the reinforcement is currently being used to examine serial pattern learning in mice and hamsters. A six-choice operant chamber has also been developed and is being used to examine the effects of drugs on rats' cognitive processes involved in serial pattern learning.

\section{Concluding Remarks}

The variations of the eight-choice circular array procedure have proven to be useful for examining a number of phenomena across a variety of species in a number of situations. These procedures have allowed for the direct comparison of the processes involved in serial pattern learning in mice, humans, and rats and could be easily adapted to examine serial pattern learning in other species. The only criterion a species needs to meet is an ability to perform numerous responses in a testing session across a number of testing days.
The procedure allows for an investigator to use a single task to evaluate a number of cognitive abilities, many in the same experiment. This task, in the past, has been used to examine rule learning (Fountain, Krauchunas, \& Rowan, 1999; Fountain \& Rowan, 1995a, 1995b; Fountain, Rowan, \& Benson, 1999), the effect of violations on rule learning (Fountain \& Rowan, 1995b, 2000), the learning of hierarchical and linear rule-based patterns (Fountain \& Rowan, 1995a), chunking of nonadjacent elements by structure (Fountain, Rowan, \& Benson, 1999), and phrasing effects (Wallace, Rowan, \& Fountain, 2001). Lastly, since serial pattern learning depends on a number of different cognitive processes working in synchronicity, this is an ideal task not only to closely examine these processes, but also to compare differences in these processes among species as well as among strains of a single species.

\section{REFERENCES}

Fountain, S. B. (1990). Rule abstraction, item memory, and chunking in rat serial-pattern tracking. Journal of Experimental Psychology: Animal Behavior Processes, 16, 96-105.

Fountain, S. B., \& Annau, Z (1984). Chunking, sorting, and rulelearning from serial patterns of brain-stimulation reward by rats. $A n$ imal Learning \& Behavior, 12, 265-274.

Fountain, S. B., Krauchunas, S. M., \& Rowan, J. D. (1999). Serialpattern learning in mice: Pattern structure and phrasing. Psychological Record, 2, 173-192.

Fountain, S. B., \& Rowan, J. D. (1995a). Coding of hierarchical versus linear pattern structure in rats and humans. Journal of Experimental Psychology: Animal Behavior Processes, 3, 187-202.

Fountain, S. B., \& Rowan, J. D. (1995b). Pattern coding in a nonverbal species: Sensitivity to violations of "runs" and "trills" structure in rats. Journal of Experimental Psychology: Animal Behavior Processes, 1, 78-81.

Fountain, S. B., \& Rowan, J. D. (2000). Differential impairments of rat serial pattern learning and retention induced by MK-801, an NMDA receptor antagonist. Psychobiology, 28, 32-44.

Fountain, S. B., Rowan, J. D., \& Benson, D. M. (1999). Rule learning in rats: Serial tracking in interleaved patterns. Animal Cognition, 2, 41-54.

Fountain, S. B., Wallace, D. G., Carman, H. M., Rowan, J. D., Stempowski, N. K., \& Benson, A. M. (in press). Discrimination learning, rule learning, and hippocampal versus nonhippocampal processes in sequential behavior. In S. B. Fountain, M. Bunsey, J. H. Danks, \& M. K. McBeath (Eds.), Animal cognition and sequential behavior: Behavioral, biological, and computational perspectives. Norwell, MA: Kluwer.

Hulse, S. H., \& DorSKy, N. P. (1977). Structural complexity as a determinant of serial pattern learning. Learning \& Motivation, 8, 488-506.

LASHLEY, K. S. (1951). The problem of serial order in behavior. In L. A. Jeffress (Ed.), Cerebral mechanisms in behavior (pp. 112-146). New York: Wiley.

Restle, F., \& Brown, E. R. (1970). Serial pattern learning: Pretraining of runs and trills. Psychonomic Science, 19, 321-322.

Restle, F., \& Burnside, B. L. (1972). Tracking of serial patterns. Journal of Experimental Psychology, 95, 299-307.

Wallace, D. G., Rowan, J. D., \& Fountain, S. B. (2001). Determinants of phrasing effects in rat serial pattern learning. Manuscript submitted for publication.

(Manuscript received October 2, 2000; revision accepted for publication May 10, 2001.) 